\title{
Correction to: Nanostructured Polymer/Nanophase Ceramic Composites Enhance Osteoblast and Chondrocyte Adhesion, by Kay S, Thapa A, Haberstroh KM, and Webster TJ. Tissue Eng Part A 8, 753, 2002.
}

\footnotetext{
TN the October 2002 issue of Tissue Engineering (vol. 8, no. 5; 753-761) in the article titled Nanostructured Polymer/ 1 Nanophase Ceramic Composites Enhance Osteoblast and Chondrocyte Adhesion by Sarina Kay, Anil Thapa, Karen M. Haberstroh, and Thomas J. Webster, the authors have become aware that the Figure 1 image in the article had previously been published in IEEE Transactions on Nanobiosciences without proper attribution.
}

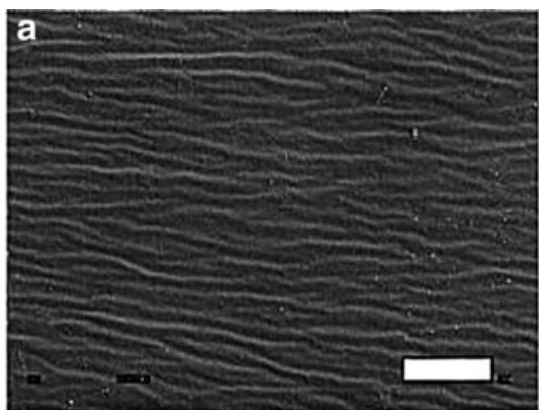

Conventional PLGA

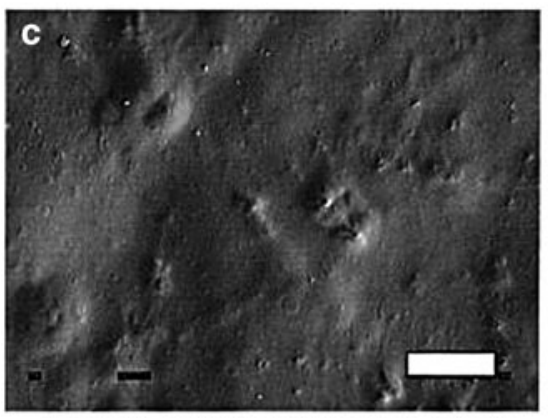

Conventional PLGA/Conventional Titania

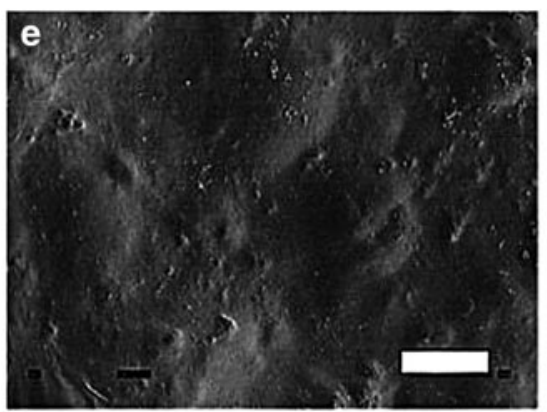

Sub-Micron PLGA/Conventional Titania

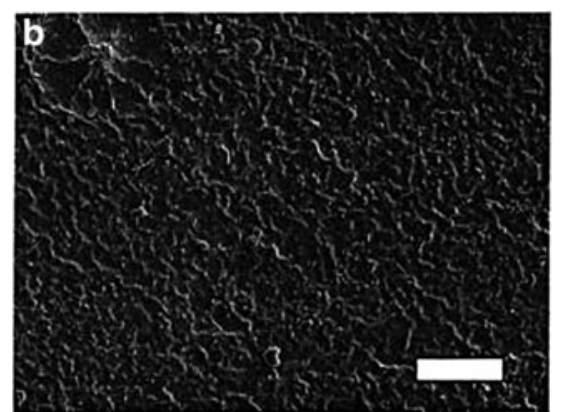

Nanophase PLGA

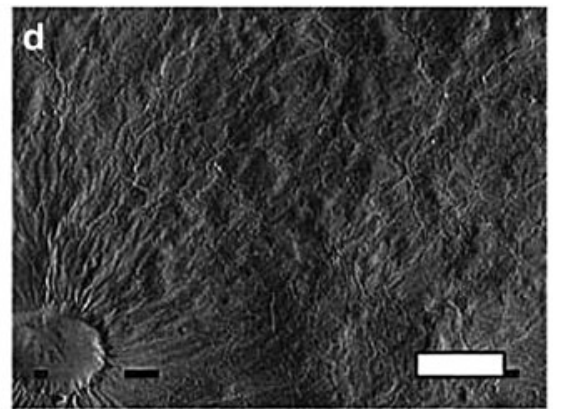

Conventional PLGA/Nanophase Titania

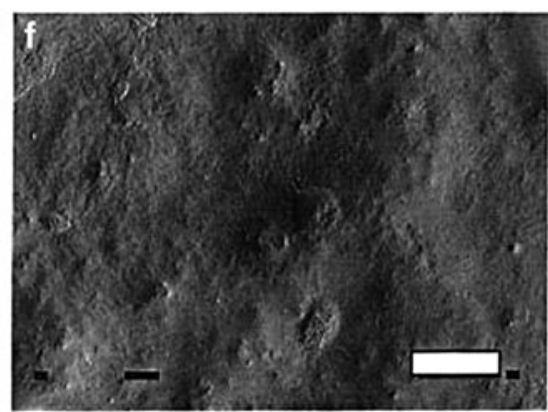

Sub-Micron PLGA/Nanophase Titania 
Both of the publications are from the authors' research group, and the image is their data. It was previously understood by the authors that the IEEE Transactions on Nanobiosciences was a conference proceeding and subsequent publications would not require permission.

The online article has been corrected to acknowledge the image having been previously published in IEEE Transactions on Nanobiosciences.

The authors apologize for the omission. 